Reprod. Nutr. Dévelop., 1984, 24 (5A), 557-561.

\title{
Sécrétion de progestérone au cours du cycle induit par l'introduction du mâle chez la chèvre créole en anœs- trus : effets de la saison
}

\author{
P. CHEMINEAU (1), Noëlle POULIN (*), Y. COGNIÉ (*)
}

Recherches zootechniques, I.N.R.A., 97170 Petit-Bourg, Guadeloupe, Antilles Françaises.

(*) Physiologie de la Reproduction, I.N.R.A., Nouzilly, 37380 Monnaie, France.

Summary. Progesterone secretion in the Creole goat during male-induced ovarian cycles : seasonal effects.

Plasma progesterone levels during male-induced ovarian cycles were measured at three periods (March, July, November) in Creole goats. For the females experiencing a cycle of normal duration, plasma progesterone reached $2.0 \mathrm{ng} / \mathrm{ml}$ six days after male introduction. For the females experiencing a short cycle, a brief (one day) and slight $(0.5$ to $1.0 \mathrm{ng} / \mathrm{ml}$ ) secretion of progesterone was observed during this cycle. A seasonal variation in the interval between male introduction and maximum level of progesterone during the short cycle was demonstrated (March : 4.7 days ; July : $5.6 \mathrm{~d}$. and November : $3.1 \mathrm{~d}$.), but the maximum level reached did not vary with season $(0.6 ; 0.9$ and $0.6 \mathrm{ng} / \mathrm{ml}$ ).

\section{Introduction.}

L'introduction du mâle parmi des femelles en repos ovarien provoque une reprise synchrone des ovulations (brebis: Oldham, Martin et Knight, 1978 ; chèvre : Shelton et Morrow, 1965 ; Chemineau, 1983) au cours des quelques jours qui suivent. Dans la moitié des cas chez la brebis (Oldham et Martin, 1978) et la majorité des cas chez la chèvre (Ott, Nelson et Hixon, 1980 ; Chemineau, 1983) le corps jaune consécutif à cette première ovulation régresse rapidement. Une nouvelle ovulation accompagnée (chèvre) ou non (brebis) d'un comportement $d^{\prime} œ$ cstrus se produit à la fin de ce cycle ovarien court ; cette nouvelle ovulation est suivie d'un cycle de durée normale. Quelle est la sécrétion de progestérone de ces corps jaunes de cycle court par rapport à celle des corps jaunes de cycle normal ? A partir de quand peut-on distinguer les cycles courts des cycles normaux?

(1) Adresse actuelle : I.N.R.A., Physiologie de la Reproduction, Nouzilly 37380 Monnaie, France. 
Avec ces objectifs, nous avons mesuré ici les taux plasmatiques de progestérone au cours des premiers jours qui ont suivi les ovulations induites par " effet bouc » chez des chèvres créoles.

\section{Matériel et méthodes.}

Aux mois de mars, juillet et novembre, respectivement 15,30 et 12 chèvres créoles (Chemineau et al., 1983) en inactivité ovarienne (jugée selon la méthode décrite par Thimonier, 1978) sont mises en présence de mâles vasectomisés (proportion : 5 à 10 mâles pour 100 femelles), après une période de complète séparation d'au moins trois semaines. Les mâles sont maintenus en permanence avec les femelles pendant toute la durée de l'expérience. Sur chaque femelle une prise de sang est réalisée quotidiennement du jour 2 au jour 8 après l'introduction des mâles, puis tous les deux jours du jour 10 au jour 13. La concentration plasmatique de progestérone est mesurée par la méthode décrite par Yenikoye et al. (1981). La sensibilité du dosage est de $5 \mathrm{pg} / \mathrm{ml}$ et le coefficient de variation intra-dosage est de $10 \%$.

Pour la construction' de la figure 1 la démarche suivante a été adoptée :

1) Chez les femelles faisant un cycle de durée normale le profil moyen de secrétion de progestérone (trois saisons confondues) est calculé par rapport au jour d'introduction du mâle.

2) Chez les femelles faisant un cycle de courte durée, la courbe moyenne par jour et par saison est d'abord calculée en considérant le pic de progestérone comme origine. Puis les courbes moyennes sont dessinées par rapport au

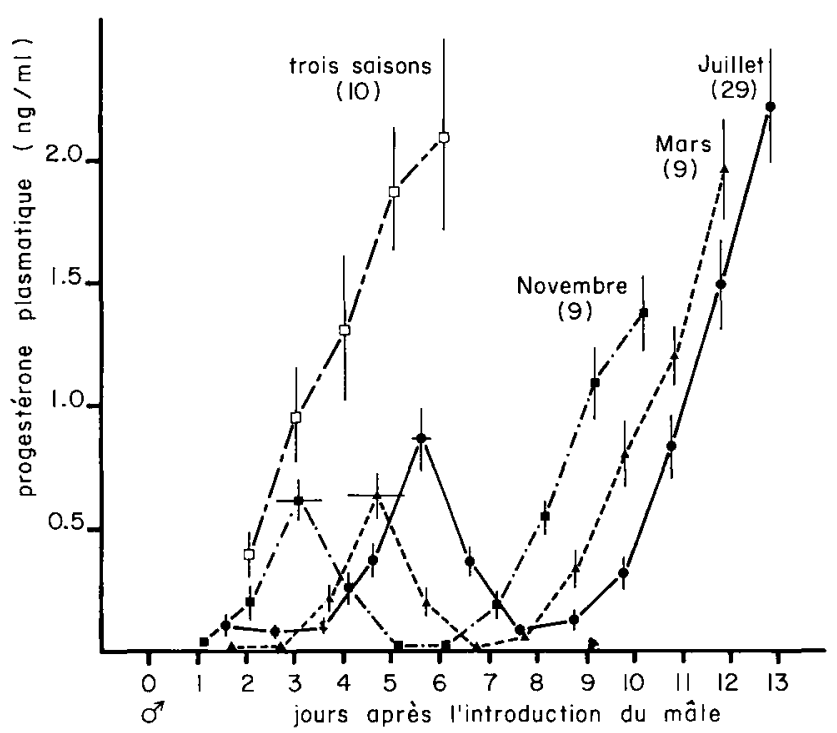

FIG. 1. - Progestérone plasmatique après l'introduction du mâle chez la chèvre créole en anoestrus (Voir texte pour la méthode de calcul des jours). Moyenne \pm erreur standard. 
moment moyen du pic de progestérone après l'introduction du mâle.

Les comparaisons statistiques sont réalisées à l'aide du test $t$ (Snedecor et Cochran, 1971).

\section{Résultats.}

A partir du deuxième jour après l'introduction du mâle, les femelles qui font un cycle de durée normale ont une concentration de progestérone plasmatique qui s'élève régulièrement depuis le niveau de base pour atteindre plus de $2,0 \mathrm{ng} / \mathrm{ml}$ le sixième jour (fig. 1). Au contraire, les femelles qui font un cycle de courte durée ont une concentration plasmatique de progestérone qui s'élève, pendant une seule journée, jusqu'à des valeurs comprises entre 0,5 et $1,0 \mathrm{ng} / \mathrm{ml}$ de plasma, puis redescend rapidement jusqu'au niveau de base (fig. 1).

Le maximum de sécrétion de progestérone au cours du cycle court induit par le mâle est observé 1,5 à 2,5 jours plus tôt en novembre qu'en juillet et qu'en mars, la différence est significative. La concentration maximale ne varie pas significativement avec la saison (tabl. 1).

\section{TABLEAU 1}

Progestéronémie au cours du cycle court induit par l'introduction du mâle en fonction de la saison chez la chèvre créole (les chiffres affectés de lettres identiques ne sont pas significativement différents au seuil de $5 \% ; m \pm$ écart-type).

\begin{tabular}{lcccc}
\hline Période & Mars & Juillet & Novembre \\
\hline Nombre de femelles & 9 & 29 & 9 \\
\hline $\begin{array}{l}\text { Intervalle introduction } \\
\text { du mâle-moment moyen du maximum } \\
\text { de progestérone (jours) }\end{array}$ & $\begin{array}{c}4,7^{\mathrm{a}} \\
\pm 1,7\end{array}$ & $\begin{array}{c}5,6^{\mathrm{a}} \\
\pm 1,2\end{array}$ & $\begin{array}{c}3,1 \mathrm{~b} \\
\pm 1,4\end{array}$ \\
\hline $\begin{array}{c}\text { Concentration maximum } \\
\text { de progestérone atteinte } \\
\text { (ng/ml de plasma) }\end{array}$ & $\begin{array}{l}0,6^{\mathrm{a}} \\
0,3\end{array}$ & $\begin{array}{l}0,9^{\mathrm{a}} \\
0,7\end{array}$ & $\begin{array}{c}0,6^{\mathrm{a}} \\
\pm 0,3\end{array}$ \\
\hline
\end{tabular}

\section{Discussion-Conclusion.}

Le corps jaune qui suit l'ovulation induite par le mâle est généralement de très courte durée (Oldham et Martin, 1978; Chemineau, 1983) ; comme le montrent les résultats présentés ici, une sécrétion transitoire de progestérone accompagne sa mise en place. Cette sécrétion, chez tous les animaux, ne dure qu'une journée et ne dépasse pas $1 \mathrm{ng} / \mathrm{ml}$ de plasma. Cette constance dans la durée du cycle ovarien induit par le mâle a déjà été décrite, après estimation de l'âge des corps jaunes par endoscopie, dans la même race (Chemineau, 1983), et chez la brebis (Oldham et Martin, 1978). Ott, Nelson et Hixon (1980), après intro- 
duction des mâles parmi des chèvres laitières, observent les mêmes profils de sécrétion de progestérone que ceux décrits ici.

Les différences saisonnières observées dans le temps écoulé entre l'introduction du mâle et le pic de progestérone sont la conséquence des moments d'ovulation variant avec la saison enregistrés sur les mêmes animaux. En effet, lorsque la date d'ovulation est appréciée par estimation visuelle de l'âge des corps jaunes, I'intervalle mâle-première ovulation varie avec "l'intensité de l'anœestrus » (Chemineau, 1983). Par contre la concentration maximale de progestérone atteinte pendant le cycle court ne varie pas avec la saison d'observation.

II semble donc que si la rapidité de la réponse ovarienne à l'introduction du mâle et la fréquence d'apparition des cycles courts sont variables (Chemineau, 1983), les caractéristiques du cycle induit sont toujours identiques. Une telle observation a également été faite chez la brebis barbarine (Khaldi, 1984). Les mécanismes qui sont à l'origine de l'apparition de ces cycles courts ou normaux restent encore inconnus. Plusieurs auteurs (Martin et al., 1980) l'attribuent au temps insuffisant de stimulation des follicules pré-ovulatoires par les hormones gonadotropes, ce qui conduirait à un mauvais développement ultérieur du tissu lutéal.

Chez les femelles manifestant un cycle de durée normale dès la première ovulation induite, la sécrétion de progestérone est très différente de ce qui est observé lors d'un cycle court puisque, 4 jours après l'introduction du mâle, la concentration de progestérone plasmatique dépasse déjà $1 \mathrm{ng} / \mathrm{ml}$. Le profil de secrétion qui est observé ici est comparable à ce qui est rapporté chez des femelles cycliques (Thorburn et Schneider, 1972 ; Chemineau et al., 1982).

Reçu en février 1984 Accepté en avril 1984

\section{Références}

CHEMINEAU P., 1983. Effect on œstrus and ovulation of exposing creole goats to the male at three times of the year. J. Reprod. Fert, 67, 65-72.

CHEMINEAU P., GAUTHIER D., POIRIER J.-C., SAUMANDE J., 1982. Plasma levels of LH, FSH, prolactin, œstradiol $17 \beta$ and progesterone during natural and induced cestrus in the dairy goat. Theriogenology, 17, 313-323.

CHEMINEAU P., COGNIE Y., XANDE A., PEROUX F., ALEXANDRE G., LEVY F., SHITALOU E., BECHE J.-M., SERGENT D., CAMUS E., BARRE N., THIMONIER J., 1983. Le cabrit créole et ses caractéristiques zootechniques : monographie. Rev. Elev. Méd. vét. Pays trop., 37 (2) (sous presse).

KHALDI G., 1984. Variations saisonnières de l'activité ovarienne, du comportement d'cestrus et de la durée de l'anoestrus post-partum des femelles de race Barbarine: influences du niveau alimentaire et de la présence du mâle. Th. Doct. Etat. Univ. Sci. Techni. du Languedoc, Montpellier II, $168 \mathrm{pp}$.

MARTIN G. B., COGNIÉ Y., GAYERIE F., OLDHAM C. M., POINDRON P., SCARAMUZZI R. J., THIERY J. C., 1980. The hormonal responses to teasing. Proc. austr. Soc. anim. Prod., 13, 77-86.

OLDHAM C. M., MARTIN G. B., 1978. Stimulation of seasonally anovular Merino ewes by rams. II. Premature regression of ram-induced corpora lutea. Anim. Reprod. Sci., 1, 291-295. 
OLDHAM C. M., MARTIN G. B., KNIGHT T. W., 1978. Stimulation of seasonally anovular Merino ewes by rams. I. Time from introduction of the rams to the preovulatory LH surge and ovulation. Anim. Reprod. Sci., 1, 283-290.

OTT R. S., NELSON D. R., HIXON J. E., 1980. Effect of presence of the male on initiation of estrous cycle activity of goats. Theriogenology, 13, 183-190.

SHELTON M., MORROW T., 1965. A study of the mechanism of male stimulation in Angora goats. Rep. Tx. agr. exp. Stn., 20-21.

SNEDECOR G. W., COCHRAN W. G., 1971. Statistical methods. 6 $6^{\text {th }}$ edn. lowa Univ. Press, Ames.

THIMONIER J., 1978. L'activité ovarienne chez les bovins. Moyens d'étude et facteurs de variations. Ann. Méd. vét., 122, 81-92.

THORBURN G. D., SCHNEIDER W., 1972. The progesterone concentration in the plasma of the goat during the oestrous cycle and pregnancy. J. Endocr., 52, 23-36.

YENIKOYE A., MARIANA J.-C., LEY J.-P., JOLIVET E., TERQUI M., LEMON-RESPLANDY M., 1981. Modèle mathématique de l'évolution de progestérone chez la vache : application et mise en évidence de différences entre races. Reprod. Nutr. Dévelop., 21, 561-575. 